\title{
La estimación de la dependencia
}

\author{
José JAVIER Silva QUINTAS \\ Actuario \\ jsilva@caser.es
}

Recibido: 21.03.2007

Aceptado: 29.05 .2007

La idea fundamental del trabajo que presentamos a continuación es la posibilidad de construir un método de cálculo basado en la creación de tablas de permanencia de un colectivo. Estas tablas tienen como característica esencial que se construyen como una función de la edad. Nuestro objetivo es mostrar un método sencillo que sirva para estimar la evolución de la situación de dependencia en España. Se trataría, por tanto, de establecer dos colectivos, moverlos en el tiempo y compararlos entre si.

Los colectivos que vamos a considerar son los siguientes:

1. La población total.

2. La población con algún grado de dependencia.

La razón para comparar estos dos colectivos y no otros, es porque nos facilitan una visión de la importancia relativa de la población dependiente y constituyen un punto de partida que nos permite tener una idea respecto a la magnitud del problema de la dependencia.

\section{DEFINICIÓN DE DEPENDENCIA}

Existen muchas definiciones del concepto de dependencia y la mayoría tiene una base común que incluye:

- Una incapacidad o limitación física o mental.

- La necesidad de ser atendido o asistido.

La definición se completaría añadiendo que la atención o asistencia se aplicaría a necesidades básicas de la vida cotidiana como pueden ser lavarse, vestirse, desplazarse, contener a voluntad orina y heces, comunicarse u otras. 
El Consejo de Europa en su Recomendación relativa a la dependencia, aprobada en septiembre de 1998 la define como «un estado en el que se encuentran las personas que por razones ligadas a la falta o la pérdida de autonomía física, psíquica o intelectual, tienen necesidad de asistencia y/o ayudas importantes a fin de realizar los actos corrientes de la vida diaria y, de modo particular, los referentes al cuidado personal».

La dependencia puede aparecer o existir a cualquier edad. De todas formas está demostrado empíricamente que existe una relación que podría asimilar las tasas de dependencia a una función cuya principal variable es la edad.

La probabilidad de aparición de la dependencia aumenta con la edad pero no de forma lineal. Existe un punto alrededor de los 80 años, en que dicho aumento crece de forma considerable.

Existen variables de tipo genético, social o ambiental relacionadas con los ratios de dependencia. Son variables sobre las que se puede actuar para reducir esos ratios (hablaríamos de prevención). Pero hay una variable sobre la que no se puede actuar, la edad.

Es sobre todo el envejecimiento de la población lo que hace que actualmente sea una de las preocupaciones básicas de un Estado del Bienestar como el nuestro. Hasta tal punto que tenemos una recién estrenada Ley que delimita y garantiza la protección de la dependencia.

\section{BASES TÉCNICAS}

Proyecciones de población. Escenario 1 de las «Proyecciones de población calculadas a partir del Censo de 2001» publicadas por el INE. Este escenario considera que las entradas netas de extranjeros en España evolucionan según la tendencia más reciente hasta el año 2010, año a partir del cual se mantienen constantes. De estos datos tomamos sólo un colectivo de partida (el del año 2002) y parte de las hipótesis de elaboración de las proyecciones, como son los nacimientos y las entradas netas del extranjero:

Hipótesis del Escenario 1

\begin{tabular}{|c|c|c|c|c|c|}
\hline Años & $\begin{array}{c}\text { Entradas } \\
\text { netas del } \\
\text { Extranjero }\end{array}$ & Nacimientos & Años & $\begin{array}{c}\text { Entradas } \\
\text { netas del } \\
\text { Extranjero }\end{array}$ & Nacimientos \\
\hline 2002 & 647.867 & 416.518 & 2032 & 273.481 & 423.255 \\
\hline 2003 & 607.961 & 439.863 & 2033 & 272.963 & 427.731 \\
\hline 2004 & 507.500 & 449.715 & 2034 & 272.445 & 432.300 \\
\hline 2005 & 460.132 & 457.971 & 2035 & 271.927 & 436.768 \\
\hline 2006 & 417.449 & 464.873 & 2036 & 271.409 & 440.957 \\
\hline 2007 & 378.983 & 470.288 & 2037 & 270.892 & 444.678 \\
\hline 2008 & 344.311 & 474.075 & 2038 & 270.374 & 447.798 \\
\hline
\end{tabular}

EMPIRIA. Revista de Metodología de Ciencias Sociales. N. ${ }^{\circ}$ 13, enero-junio, 2007, pp. 143-161. ISSN: 1139-5737 


\begin{tabular}{|c|c|c|c|c|c|}
\hline Años & $\begin{array}{c}\text { Entradas } \\
\text { netas del } \\
\text { Extranjero }\end{array}$ & Nacimientos & Años & $\begin{array}{c}\text { Entradas } \\
\text { netas del } \\
\text { Extranjero }\end{array}$ & Nacimientos \\
\hline 2009 & 313.056 & 477.941 & 2039 & 269.856 & 450.248 \\
\hline 2010 & 284.874 & 479.800 & 2040 & 269.338 & 451.989 \\
\hline 2011 & 284.356 & 479.820 & 2041 & 268.820 & 453.008 \\
\hline 2012 & 283.838 & 478.286 & 2042 & 268.302 & 453.316 \\
\hline 2013 & 283.320 & 475.397 & 2043 & 267.784 & 452.936 \\
\hline 2014 & 282.802 & 471.462 & 2044 & 267.267 & 451.906 \\
\hline 2015 & 282.284 & 466.868 & 2045 & 266.749 & 450.272 \\
\hline 2016 & 281.767 & 462.043 & 2046 & 266.231 & 448.094 \\
\hline 2017 & 281.249 & 451.893 & 2047 & 265.713 & 445.447 \\
\hline 2018 & 280.731 & 442.466 & 2048 & 265.195 & 442.436 \\
\hline 2019 & 280.213 & 434.029 & 2049 & 264.677 & 439.172 \\
\hline 2020 & 279.695 & 426.724 & 2050 & 264.159 & 435.767 \\
\hline 2021 & 279.177 & 420.625 & 2051 & 263.642 & 432.358 \\
\hline 2022 & 278.659 & 415.750 & 2052 & 263.124 & 429.084 \\
\hline 2023 & 278.142 & 412.064 & 2053 & 262.606 & 426.064 \\
\hline 2024 & 277.624 & 409.520 & 2054 & 262.088 & 423.389 \\
\hline 2025 & 277.106 & 408.045 & 2055 & 261.570 & 421.160 \\
\hline 2026 & 276.588 & 407.603 & 2056 & 261.052 & 419.425 \\
\hline 2027 & 276.070 & 408.161 & 2057 & 260.534 & 418.217 \\
\hline 2028 & 275.552 & 409.665 & 2058 & 260.017 & 417.539 \\
\hline 2029 & 275.034 & 412.063 & 2059 & 259.499 & 417.370 \\
\hline 2030 & 274.517 & 415.253 & & & \\
\hline 2031 & 273.999 & 419.123 & & & \\
\hline
\end{tabular}

Con objeto de delimitar la fiabilidad de los datos contenidos en la tabla anterior se extrae un párrafo sobre la metodología utilizada por el INE para su construcción: «El considerable aumento que han registrado las cifras de inmigrantes en España, durante los últimos años, y la diversidad de factores que influyen en su trayectoria, suponen un notable grado de incertidumbre sobre el devenir de los correspondientes flujos de entradas y de salidas, lo que ha aconsejado el cálculo de proyecciones bajo dos supuestos distintos sobre su devenir. Los resultados correspondientes constituyen escenarios, que ilustran las consecuencias, en el crecimiento y en la distribución por edades de la población, de los distintos supuestos».

El mencionado como segundo escenario, «que supone a medio plazo una disminución mayor de las entradas netas, se ha establecido de acuerdo con Eurostat. Las entradas netas del extranjero son las mismas que en el Escenario 1 para el período 2002-2006 y, a partir de este último año, la tendencia decreciente de las entradas de extranjeros hasta el año 2010, las sitúa en torno a las 100.000 anuales para el periodo 2007-2059, por lo que el total de entradas durante el mismo es de unos 5,8 millones de personas». 
Las hipótesis relacionadas con la mortalidad en este escenario 1 utilizado no se toman en consideración, y son sustituidas por otras.

Tablas de mortalidad. PERM/F2000P tablas generacionales utilizadas en el sector asegurador. Hay que matizar que están basadas en estadísticas de asegurados facilitadas por UNESPA, y no del total de población, lo cual limita la validez para un estudio de mortalidad en el total de la población. Sin embargo tienen una ventaja obre otras tablas susceptibles de ser utilizadas y es que las PERM/F2000P son tablas dinámicas que tienen en cuenta que la esperanza de vida no solo depende de edad y sexo sino también de la generación a la que pertenece. Una persona nacida en 1960 tiene una esperanza de vida al nacer inferior a la que tiene otra persona nacida en el año 2006.

Para más información sobre estas tablas se recomienda la lectura de «PROYECCION Y ESTUDIO DE UNA POBLACION. EL PAPEL DE LA MORTALIDAD» de Ana de Vicente Merino, Julio Hernández March, Irene Albarrán Lozano y Cruz Ramírez Pérez (Universidad Complutense de Madrid)

A efectos de este estudio el proceso de cálculo para la generación de estas tablas se considera siempre el mismo y es el descrito en la «Resolución de 3 de octubre de 2000 de la Dirección General de Seguros y Fondos de Pensiones por la que se da cumplimiento a lo previsto en el número 5 de la disposición transitoria segunda del Reglamento de Ordenación y supervisión de los seguros privados, aprobado por Real Decreto 2486/1998, de 20 de noviembre, en relación con las tablas de mortalidad y supervivencia a utilizar por las entidades aseguradoras».

Las «Tablas de mortalidad de la población de España 1998-1999» publicadas por el INE se utilizarán para establecer algunos ratios de partida.

Ratios de dependencia. Se calculan con la comparación del número de discapacitados extraídos de la «Encuesta de Discapacidades, Deficiencias y Estado de Salud 1999» y el total de población según la «Población nacional de derecho calculada a 15 de mayo de 1999» como resultado de la «Revisión de las Proyecciones de Población en base al Censo de Población de 1991» Todos ellos datos publicados por el INE.

Razones de salida del colectivo de discapacitados. no se consideran, salvo el fallecimiento.

Distribución de nacimientos por sexo. Para simplificar y puesto que el efecto es irrelevante se considera la misma probabilidad de nacer varón que de nacer mujer.

Distribución de entradas del extranjero por edad y sexo. Se estiman a partir del cálculo de las diferencias entre las «Proyecciones de población calculadas a partir del Censo de 2001» y las calculadas partiendo de los datos del año 2002 aplicando las tasas de mortalidad de las «Tablas de mortalidad de la población de 
España 1998-1999» publicadas por el INE. Los ratios quedan como sigue:

\begin{tabular}{ccc}
\hline Edad & Varones & Mujeres \\
\hline 0 años & $1,58 \%$ & $0,00 \%$ \\
1 años & $1,13 \%$ & $1,03 \%$ \\
2 años & $0,00 \%$ & $0,00 \%$ \\
3 años & $0,00 \%$ & $0,00 \%$ \\
4 años & $1,00 \%$ & $0,00 \%$ \\
5 años & $0,99 \%$ & $0,00 \%$ \\
6 años & $0,99 \%$ & $0,00 \%$ \\
7 años & $0,97 \%$ & $0,00 \%$ \\
8 años & $0,00 \%$ & $0,00 \%$ \\
9 años & $0,00 \%$ & $0,00 \%$ \\
10 años & $0,00 \%$ & $0,00 \%$ \\
11 años & $0,00 \%$ & $0,00 \%$ \\
12 años & $0,00 \%$ & $0,00 \%$ \\
13 años & $0,00 \%$ & $0,00 \%$ \\
14 años & $0,00 \%$ & $0,00 \%$ \\
15 años & $0,00 \%$ & $0,00 \%$ \\
16 años & $0,00 \%$ & $0,00 \%$ \\
17 años & $0,00 \%$ & $0,00 \%$ \\
18 años & $1,11 \%$ & $1,12 \%$ \\
19 años & $1,34 \%$ & $1,46 \%$ \\
20 años & $1,64 \%$ & $1,74 \%$ \\
21 años & $1,93 \%$ & $1,97 \%$ \\
22 años & $2,20 \%$ & $2,16 \%$ \\
\hline
\end{tabular}

\begin{tabular}{ccc}
\hline Edad & Varones & Mujeres \\
\hline 23 años & $2,41 \%$ & $2,34 \%$ \\
24 años & $2,61 \%$ & $2,45 \%$ \\
25 años & $2,74 \%$ & $2,45 \%$ \\
26 años & $2,76 \%$ & $2,39 \%$ \\
27 años & $2,74 \%$ & $2,27 \%$ \\
28 años & $2,66 \%$ & $2,15 \%$ \\
29 años & $2,55 \%$ & $2,00 \%$ \\
30 años & $2,44 \%$ & $1,85 \%$ \\
31 años & $2,28 \%$ & $1,77 \%$ \\
32 años & $2,15 \%$ & $1,68 \%$ \\
33 años & $2,07 \%$ & $1,58 \%$ \\
34 años & $1,97 \%$ & $1,50 \%$ \\
35 años & $1,86 \%$ & $1,46 \%$ \\
36 años & $1,71 \%$ & $1,35 \%$ \\
37 años & $1,54 \%$ & $1,23 \%$ \\
38 años & $1,43 \%$ & $1,16 \%$ \\
39 años & $1,36 \%$ & $1,10 \%$ \\
40 años & $1,27 \%$ & $1,07 \%$ \\
41 años & $1,19 \%$ & $1,00 \%$ \\
42 años & $1,11 \%$ & $0,00 \%$ \\
43 años & $1,05 \%$ & $0,00 \%$ \\
44 años & $0,99 \%$ & $0,00 \%$ \\
$>44$ años & $0,00 \%$ & $0,00 \%$ \\
\hline
\end{tabular}

Activos por sexo y grupo de edad por trimestres para el año 2005 de la Encuesta de población activa publicada por el INE

Ocupados por sexo y grupo de edad por trimestres para el año 2005 de la Encuesta de población activa publicada por el INE

\section{EVOLUCION DE LA POBLACIÓN TOTAL}

Se hace una estimación de la evolución de la población en España, partiendo de la proyección de población a 2002 calculada según el escenario 1 de las «Proyecciones de población calculadas a partir del Censo de 2001» publicadas por el INE. En ningún caso se pretende rehacer los cálculos del INE, sino llegar a otros paralelos que se obtengan como resultado de la aplicación de las bases técnicas mencionadas en el punto anterior. En particular es significativo el efecto de la mortalidad considerada. Como se verá, la utilización de las tablas PERM/F2000P da como resultado una población mayor en número y más envejecida que la proyectada por el INE. 
Población por edad y sexo para el año 2002

\begin{tabular}{|c|c|c|c|c|c|}
\hline \multicolumn{3}{|c|}{ Sexo } & \multicolumn{3}{|c|}{ Sexo } \\
\hline Edad & Varones & Mujeres & Edad & Varones & Mujeres \\
\hline 0 años & 210.063 & 200.405 & 51 años & 246.313 & 250.400 \\
\hline 1 años & 204.857 & 192.558 & 52 años & 241.586 & 246.794 \\
\hline 2 años & 196.596 & 186.573 & 53 años & 238.203 & 244.572 \\
\hline 3 años & 191.654 & 181.148 & 54 años & 238.206 & 245.935 \\
\hline 4 años & 189.228 & 180.057 & 55 años & 233.968 & 242.543 \\
\hline 5 años & 190.098 & 180.511 & 56 años & 226.911 & 236.083 \\
\hline 6 años & 191.954 & 182.265 & 57 años & 218.289 & 228.110 \\
\hline 7 años & 195.849 & 185.850 & 58 años & 209.013 & 218.826 \\
\hline 8 años & 200.165 & 189.447 & 59 años & 205.513 & 216.127 \\
\hline 9 años & 204.514 & 193.579 & 60 años & 189.423 & 200.790 \\
\hline 10 años & 209.267 & 198.067 & 61 años & 177.850 & 190.502 \\
\hline 11 años & 213.073 & 201.666 & 62 años & 176.499 & 190.952 \\
\hline 12 años & 216.108 & 204.381 & 63 años & 181.151 & 199.476 \\
\hline 13 años & 220.557 & 208.859 & 64 años & 176.678 & 197.096 \\
\hline 14 años & 226.408 & 214.498 & 65 años & 186.852 & 209.720 \\
\hline 15 años & 233.292 & 221.080 & 66 años & 193.631 & 219.243 \\
\hline 16 años & 241.324 & 229.038 & 67 años & 195.326 & 223.561 \\
\hline 17 años & 251.953 & 239.553 & 68 años & 191.618 & 220.599 \\
\hline 18 años & 264.562 & 251.602 & 69 años & 188.033 & 219.984 \\
\hline 19 años & 278.433 & 265.297 & 70 años & 182.433 & 216.736 \\
\hline 20 años & 292.991 & 279.663 & 71 años & 174.860 & 211.723 \\
\hline 21 años & 309.762 & 295.605 & 72 años & 165.419 & 204.293 \\
\hline 22 años & 324.765 & 309.624 & 73 años & 157.369 & 198.925 \\
\hline 23 años & 338.431 & 323.055 & 74 años & 147.473 & 190.718 \\
\hline 24 años & 348.996 & 332.843 & 75 años & 138.573 & 183.919 \\
\hline 25 años & 356.493 & 340.177 & 76 años & 129.039 & 175.357 \\
\hline 26 años & 358.731 & 342.637 & 77 años & 120.523 & 169.179 \\
\hline 27 años & 359.293 & 343.741 & 78 años & 110.550 & 159.315 \\
\hline 28 años & 356.926 & 341.690 & 79 años & 100.057 & 149.811 \\
\hline 29 años & 353.591 & 339.041 & 80 años & 87.919 & 137.031 \\
\hline 30 años & 349.974 & 336.090 & 81 años & 76.160 & 125.396 \\
\hline 31 años & 347.167 & 334.414 & 82 años & 64.876 & 112.124 \\
\hline 32 años & 345.003 & 333.657 & 83 años & 54.623 & 100.891 \\
\hline 33 años & 342.877 & 332.862 & 84 años & 46.604 & 90.008 \\
\hline 34 años & 340.968 & 332.761 & 85 años & 40.691 & 82.185 \\
\hline 35 años & 341.029 & 334.383 & 86 años & 35.241 & 73.462 \\
\hline 36 años & 338.443 & 332.862 & 87 años & 30.255 & 65.455 \\
\hline 37 años & 333.030 & 328.657 & 88 años & 25.624 & 57.332 \\
\hline 38 años & 327.249 & 324.359 & 89 años & 21.192 & 49.334 \\
\hline 39 años & 322.586 & 320.600 & 90 años & 17.131 & 41.100 \\
\hline 40 años & 315.003 & 313.952 & 91 años & 13.517 & 33.548 \\
\hline 41 años & 309.314 & 309.350 & 92 años & 10.393 & 26.572 \\
\hline 42 años & 304.586 & 305.361 & 93 años & 7.772 & 20.707 \\
\hline 43 años & 297.278 & 298.400 & 94 años & 5.662 & 15.572 \\
\hline 44 años & 287.823 & 289.427 & 95 años & 4.014 & 11.525 \\
\hline 45 años & 277.637 & 279.837 & 96 años & 2.783 & 8.342 \\
\hline 46 años & 269.044 & 271.441 & 97 años & 1.866 & 5.903 \\
\hline 47 años & 261.807 & 263.988 & 98 años & 1.062 & 3.505 \\
\hline 48 años & 254.932 & 257.340 & 99 años & 686 & 2.452 \\
\hline 49 años & 248.635 & 251.180 & 100 y más & 1.447 & 3.905 \\
\hline 50 años & 246.423 & 249.556 & & & \\
\hline
\end{tabular}

EMPIRIA. Revista de Metodología de Ciencias Sociales. N. ${ }^{\circ}$ 13, enero-junio, 2007, pp. 143-161. ISSN: 1139-5737 
Los cálculos de las sucesivas tablas de población se realizan mediante la siguiente formulación:

\section{Edad cero}

Sea:

$\mathrm{L}(\mathrm{e}, \mathrm{m}, \mathrm{s})$ : número de personas de edad e, en el año $\mathrm{m}$ y de sexo $\mathrm{s}$

Se toma como valor:

$\mathrm{L}(0, \mathrm{~m}, \mathrm{~s})=$ número de personas para la edad 0 y sexo $\mathrm{s}$ en el año $\mathrm{m}$ de las tablas de «Proyecciones de población calculadas a partir del Censo de 2001»

\section{$100>$ Edad $>0$}

$$
\begin{aligned}
& \mathrm{L}(\mathrm{e}, \mathrm{m}, \mathrm{s})=\mathrm{L}(\mathrm{e}-1, \mathrm{~m}-1, \mathrm{~s}) \times \mathrm{p}_{\mathrm{e}-1}+\mathrm{I}(\mathrm{e}, \mathrm{m}, \mathrm{s}) \\
& \mathrm{I}(\mathrm{e}, \mathrm{m}, \mathrm{s})=\mathrm{I}(\mathrm{m}) \times \mathrm{DI}(\mathrm{e}, \mathrm{s})
\end{aligned}
$$

Donde:

$\mathrm{p}_{\mathrm{e}-1}$ : Probabilidad de que una persona de edad e- 1 alcance la edad e

$\mathrm{I}(\mathrm{e}, \mathrm{m}, \mathrm{s})$ : número de inmigrantes en el año $\mathrm{m}$, de edad e y sexo $\mathrm{s}$. No se considera mortalidad en el año de entrada: se entiende incluida en los valores netos de inmigración. Las entradas netas del extranjero se consideran sólo como entradas: para simplificar, las salidas son cero.

I(m): entradas netas del extranjero en el año m según las hipótesis del escenario 1

DI $(e, s)$ : porcentaje de personas de edad e y sexo s según la tabla de «Distribución de entradas del extranjero por edad y sexo» que aparece en las bases técnicas.

\section{Edad $>99$}

$$
\mathrm{L}(100, \mathrm{~m}, \mathrm{~s})=[\mathrm{L}(99, \mathrm{~m}-1, \mathrm{~s})+\mathrm{L}(100, \mathrm{~m}-1, \mathrm{~s})] \times \mathrm{p}_{>99}
$$

En este caso:

$\mathrm{p}_{>99}=$ probabilidad de que una persona de 99 o más años alcance un año más de edad con vid

\section{Pirámides de edad}

Con las formulaciones anteriores se llega a construir unas tablas de población por edad y sexo que, como ejemplo, representamos con tres pirámides de edad para los años: 2002 (como origen) y 2025. 

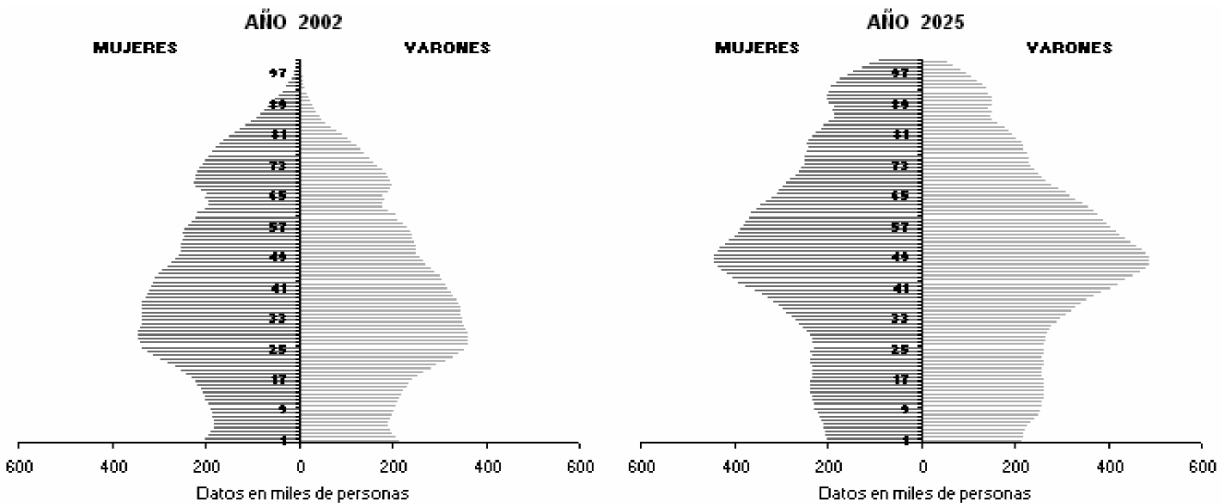

Observando las anteriores pirámides y sin necesidad de hacer cálculo alguno se puede ver el envejecimiento de la población, comprobando el desplazamiento del grueso de la pirámide hacia su parte alta.

\section{TASAS DE DEPENDENCIA}

Para la estimación de la evolución de la población dependiente se necesitan ratios de dependencia, entendidos como el porcentaje de personas que con una edad concreta pasan a tener algún grado de dependencia.

Los datos de que se dispone son, por un lado el número de personas con algún grado de dependencia por tramos de edad y sexo según encuesta del año 1999, por otro lado la población nacional de derecho calculada a 15 de mayo de 1999. Con ellos se puede calcular el porcentaje de personas dependientes por tramos de edad.

\begin{tabular}{l|rr|rr|rr}
\hline & \multicolumn{2}{|c|}{$\begin{array}{c}\text { Número de } \\
\text { dependientes }\end{array}$} & \multicolumn{2}{c|}{$\begin{array}{c}\text { Población } \\
\text { española }\end{array}$} & \multicolumn{2}{c}{$\begin{array}{c}\text { Dependientes/ } \\
\text { Población }\end{array}$} \\
\hline \multicolumn{1}{c|}{ Tramo edad } & Varones & Mujeres & Varones & Mujeres & Varones & Mujeres \\
\hline De 6 a 16 años & 43.050 & 37.210 & 2.418 .917 & 2.289 .654 & $1,78 \%$ & $1,63 \%$ \\
De 17 a 24 años & 57.453 & 33.908 & 2.510 .936 & 2.403 .985 & $2,29 \%$ & $1,41 \%$ \\
De 25 a 34 años & 113.092 & 72.814 & 3.317 .394 & 3.215 .323 & $3,41 \%$ & $2,26 \%$ \\
De 35 a 44 años & 121.191 & 109.060 & 2.872 .971 & 2.867 .990 & $4,22 \%$ & $3,80 \%$ \\
De 45 a 54 años & 141.183 & 164.726 & 2.360 .206 & 2.401 .800 & $5,98 \%$ & $6,86 \%$ \\
De 55 a 64 años & 238.469 & 273.835 & 1.900 .686 & 2.043 .129 & $12,55 \%$ & $13,40 \%$ \\
De 65 a 69 años & 161.083 & 225.255 & 942.198 & 1.086 .712 & $17,10 \%$ & $20,73 \%$ \\
De 70 a 74 años & 168.643 & 288.626 & 768.251 & 964.027 & $21,95 \%$ & $29,94 \%$ \\
De 75 a 79 años & 172.670 & 304.256 & 531.038 & 762.259 & $32,52 \%$ & $39,92 \%$ \\
De 80 a 84 años & 120.382 & 250.319 & 280.653 & 499.911 & $42,89 \%$ & $50,07 \%$ \\
De 85 y más años & 111.031 & 270.388 & 187.666 & 411.809 & $59,16 \%$ & $65,66 \%$ \\
\hline
\end{tabular}

EMPIRIA. Revista de Metodología de Ciencias Sociales. N. ${ }^{\circ}$ 13, enero-junio, 2007, pp. 143-161. ISSN: 1139-5737 
En un principio los resultados de la encuesta pretendían ajustarse lo más posible a la «Clasificación Internacional de Deficiencias, Discapacidades y Minusvalías» (CIDDM) propuesta en 1980 por la Organización Mundial de la Salud. La clasificación de las deficiencias utiliza una terminología médica que presenta una gran complejidad lo que dificulta la obtención de respuesta por parte de la población objeto de estudio.

Según se puede leer en el Libro Blanco, para operativizar la definición de discapacidad adoptada por la encuesta («una limitación de la capacidad humana que hace imposible o dificulta gravemente la actividad normal de la persona»), se estableció un listado de actividades básicas que permitiera preguntar a los encuestados de 6 y más años cuál o cuáles de ellas era incapaz de o tenía dificultades para realizar. Esas actividades básicas se agrupan, a su vez, en categorías que se corresponden con los grupos de discapacidades sobre los que aporta información la encuesta. En concreto, se han contemplado 36 actividades agrupadas en diez categorías, de acuerdo con el esquema siguiente:

\section{Ver}

1.1. Percibir cualquier imagen

1.2. Realizar tareas visuales de conjunto

1.3. Realizar tareas visuales de detalle

1.4. Ver en condiciones de iluminación pobres, diferenciar colores, etc.

2. Oír

2.1. Recibir cualquier sonido

2.2. Audición de sonidos fuertes

2.3. Escuchar el habla

3. Comunicarse

3.1. Comunicarse a través del habla

3.2. Comunicarse a través de lenguajes alternativos

3.3. Comunicarse a través de gestos no signados

3.4. Comunicarse a través de escritura-lectura convencional

4. Aprender, aplicar conocimientos y desarrollar tareas

4.1. Reconocer personas y objetos y orientarse

4.2. Recordar informaciones y episodios

4.3. Entender y ejecutar órdenes y/o tareas sencillas

4.4. Entender y ejecutar órdenes y/o tareas complejas 
5. Desplazarse

5.1. Cambiar y mantener las posiciones del cuerpo

5.2. Levantarse, acostarse

5.3. Desplazarse dentro del hogar

6. Utilizar brazos y manos

6.1. Trasladar objetos no muy pesados

6.2. Utilizar utensilios y herramientas

6.3. Manipular objetos pequeños con manos y dedos

7. Desplazarse fuera del hogar

7.1. Deambular sin medio de transporte

7.2. Desplazarse en transporte público

7.3. Conducir vehículo propio (entre 18 y 75 años)

8. Cuidar de sí mismo

8.1. Asearse solo: lavarse y cuidar su aspecto

8.2. Controlar las necesidades y utilizar solo el servicio

8.3. Vestirse-desvestirse y arreglarse

8.4. Comer y beber

9. Realizar las tareas del hogar (mayores de 10 años)

9.1. Hacer compras y controlar los suministros y servicios

9.2. Cuidarse de las comidas

9.3. Limpieza y cuidado de la ropa

9.4. Limpieza y mantenimiento de la casa

9.5. Cuidarse del bienestar del resto de la familia

10. Relacionarse con otras personas

10.1. Mantener relaciones de cariño con familiares

10.2. Hacer nuevos amigos y mantener la amistad

10.3. Relacionarse con compañeros, jefes, etc.

Por tanto, los porcentajes apuntados en el cuadro al principio de este apartado están condicionados por la elección de estas 36 actividades.

En los presentes cálculos las personas dependientes tienen la misma mortalidad que las que no lo son. Es decir, se utilizan tablas generacionales en las que la mortalidad mejora a aumentar el año de nacimiento. Esto hace que los porcentajes del cuadro anterior se modifiquen con el paso del tiempo, porque el número de personas dependientes a una determinada edad depende de la personas que alcanzan ese estado en el año más las que siendo dependientes con anterioridad sobreviven hasta esa edad (la cuantía de estas últimas depende de la mortalidad utilizada). 
Lo que se pretende ahora es conseguir los mismos porcentajes para cada edad. Eso se consigue creando una función que se aproxime lo suficiente a los datos calculados.

\section{Ajustes a una función}

Los anteriores tramos de edad se convierten en edades concretas utilizando medias de edad de la siguiente forma:

$$
\mathrm{e}_{\mathrm{ij}}=\sum_{\mathrm{e}=\mathrm{i}}^{\mathrm{j}} \operatorname{Pob}_{\mathrm{e}} \mathrm{e} / \sum_{\mathrm{e}=\mathrm{i}}^{\mathrm{j}} \operatorname{Pob}_{\mathrm{e}}
$$

Donde:

$\mathrm{e}_{\mathrm{ij}}$ : edad representativa del tramo de edades que va desde la edad i a la $\mathrm{j}$ $\mathrm{Pob}_{\mathrm{e}}$ : número de personas con edad e según el cuadro de edades para el año 2002 que parece anteriormente

e: edad que tomará los valores desde i hasta $\mathrm{j}$

Este cálculo se hace para cada sexo.

La función a la que se pretende ajustar los datos así obtenidos es del tipo:

$$
\% \mathrm{D}_{\mathrm{e}}=\mathrm{a}+\mathrm{b} \times \mathrm{e}^{\mathrm{c}}
$$

Donde:

$\mathrm{a}, \mathrm{b}, \mathrm{c}$ : constantes

$\% \mathrm{D}_{\mathrm{e}}$ : porcentaje de personas dependientes con edad e sobre el total de personas de esa edad

e: edad

Los valores obtenidos para las constantes a, b y c son:

\begin{tabular}{ccc}
\hline & Varones & Mujeres \\
\hline a & 0,02095 & 0,01123 \\
b & $9,8785 \mathrm{E}-10$ & $1,0613 \mathrm{E}-08$ \\
c & 4,5 & 4 \\
\hline
\end{tabular}

En los siguientes gráficos se puede comprobar la bondad de las funciones obtenidas. 


\section{VARONES}

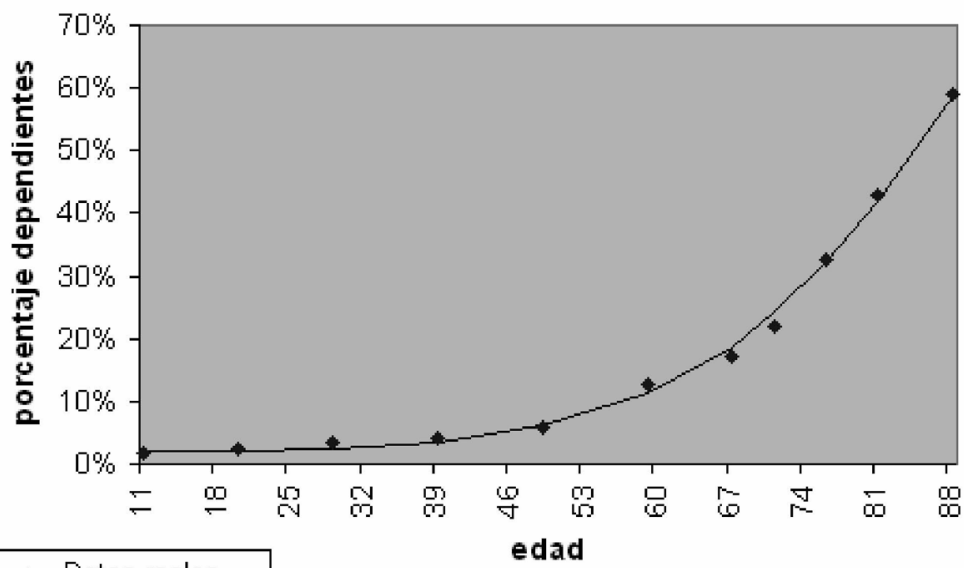

- Datos reales

Ajuste a función

\section{MUJERES}

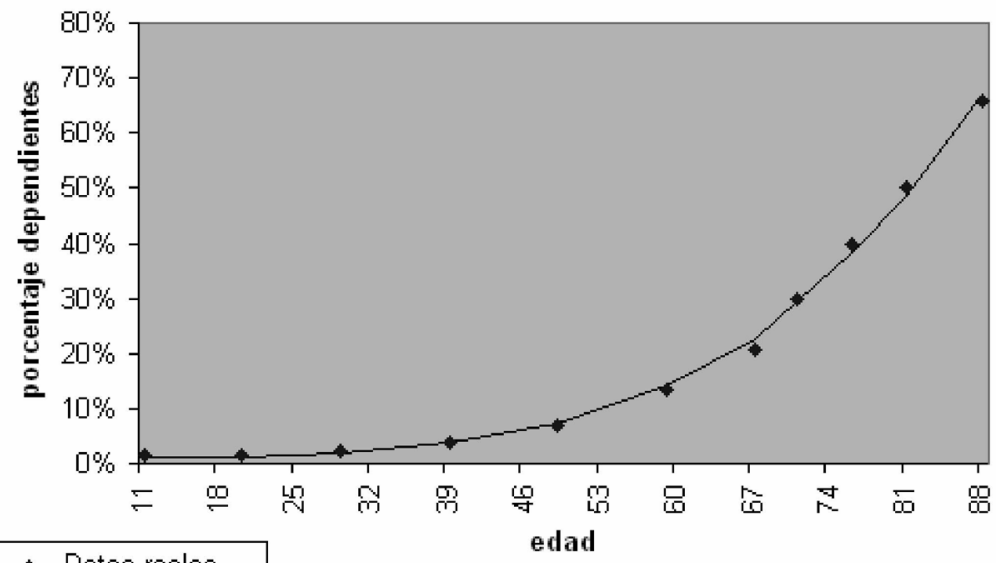

- Datos reales Ajuste a función

Es estos casos el coeficiente de determinación es:

$$
\begin{aligned}
& \mathrm{R} 2=0,9967 \text { para hombres } \\
& \mathrm{R} 2=0,9976 \text { para mujeres } \hat{\mathrm{Y}}
\end{aligned}
$$




\section{Tasas de dependencia}

Se calculan unas tasas que representan la probabilidad de que una persona de una determinada edad que no es dependiente alcance algún grado de dependencia antes de cumplir un años más.

Para ello se toman como base las «Tablas de mortalidad de la población de España 1998-1999» publicadas por el INE definidas por las columnas L(x), que representan el número de personas que, partiendo de una edad cero con un importe de L(0), alcanzan la edad x.

Se crea una tabla de personas activas, consideradas estas como las que no tienen algún grado de dependencia.

$$
\begin{aligned}
& \mathrm{La}(0)=\mathrm{L}(0) \\
& \mathrm{La}(\mathrm{e})=\left[\mathrm{La}(\mathrm{e}-1)-\mathrm{L}(\mathrm{e}-1) \times \% \mathrm{D}_{\mathrm{e}-1}\right] \times \mathrm{L}(\mathrm{e}) / \mathrm{L}(\mathrm{e}-1)
\end{aligned}
$$

Siendo:

La(e): número de personas que, partiendo de un colectivo inicial de L(0) alcanzan la edad e sin algún grado de dependencia

$\mathrm{L}(\mathrm{e})$ : valor de $\mathrm{L}(\mathrm{x})$ de la tabla de mortalidad de la población de España 1998-1999 para la edad e.

Las tasas de dependencia se calculan sobre la población activa de la siguiente forma:

$$
\mathrm{d}_{\mathrm{e}}=\mathrm{L}(\mathrm{e}) \times \% \mathrm{D}_{\mathrm{e}} / \mathrm{La}(\mathrm{e})
$$

donde $\mathrm{d}_{\mathrm{e}}$ es la tasa buscada para la edad e.

En la siguiente tabla se pueden ver los resultados de estos cálculos. 


\begin{tabular}{|c|c|c|c|c|c|}
\hline \multicolumn{3}{|c|}{ Tasas de dependencia } & \multicolumn{3}{|c|}{ Tasas de dependencia } \\
\hline Edad & Varones & Mujeres & Edad & Varones & Mujeres \\
\hline 0 & $2,0948 \%$ & $1,1234 \%$ & 51 & $0,4350 \%$ & $0,5928 \%$ \\
\hline 1 & $0,0000 \%$ & $0,0000 \%$ & 52 & $0,4679 \%$ & $0,6324 \%$ \\
\hline 2 & $0,0000 \%$ & $0,0000 \%$ & 53 & $0,5029 \%$ & $0,6742 \%$ \\
\hline 3 & $0,0000 \%$ & $0,0001 \%$ & 54 & $0,5399 \%$ & $0,7183 \%$ \\
\hline 4 & $0,0000 \%$ & $0,0002 \%$ & 55 & $0,5792 \%$ & $0,7649 \%$ \\
\hline 5 & $0,0001 \%$ & $0,0004 \%$ & 56 & $0,6208 \%$ & $0,8140 \%$ \\
\hline 6 & $0,0002 \%$ & $0,0007 \%$ & 57 & $0,6650 \%$ & $0,8658 \%$ \\
\hline 7 & $0,0003 \%$ & $0,0012 \%$ & 58 & $0,7119 \%$ & $0,9206 \%$ \\
\hline 8 & $0,0005 \%$ & $0,0018 \%$ & 59 & $0,7615 \%$ & $0,9785 \%$ \\
\hline 9 & $0,0008 \%$ & $0,0026 \%$ & 60 & $0,8143 \%$ & $1,0397 \%$ \\
\hline 10 & $0,0012 \%$ & $0,0037 \%$ & 61 & $0,8703 \%$ & $1,1044 \%$ \\
\hline 11 & $0,0017 \%$ & $0,0050 \%$ & 62 & $0,9298 \%$ & $1,1731 \%$ \\
\hline 12 & $0,0023 \%$ & $0,0065 \%$ & 63 & $0,9930 \%$ & $1,2459 \%$ \\
\hline 13 & $0,0031 \%$ & $0,0084 \%$ & 64 & $1,0603 \%$ & $1,3231 \%$ \\
\hline 14 & $0,0041 \%$ & $0,0106 \%$ & 65 & $1,1319 \%$ & $1,4052 \%$ \\
\hline 15 & $0,0053 \%$ & $0,0131 \%$ & 66 & $1,2081 \%$ & $1,4925 \%$ \\
\hline 16 & $0,0067 \%$ & $0,0160 \%$ & 67 & $1,2895 \%$ & $1,5856 \%$ \\
\hline 17 & $0,0083 \%$ & $0,0193 \%$ & 68 & $1,3764 \%$ & $1,6849 \%$ \\
\hline 18 & $0,0102 \%$ & $0,0230 \%$ & 69 & $1,4693 \%$ & $1,7911 \%$ \\
\hline 19 & $0,0124 \%$ & $0,0272 \%$ & 70 & $1,5688 \%$ & $1,9048 \%$ \\
\hline 20 & $0,0149 \%$ & $0,0319 \%$ & 71 & $1,6756 \%$ & $2,0268 \%$ \\
\hline 21 & $0,0177 \%$ & $0,0371 \%$ & 72 & $1,7902 \%$ & $2,1580 \%$ \\
\hline 22 & $0,0210 \%$ & $0,0428 \%$ & 73 & $1,9137 \%$ & $2,2995 \%$ \\
\hline 23 & $0,0246 \%$ & $0,0491 \%$ & 74 & $2,0468 \%$ & $2,4523 \%$ \\
\hline 24 & $0,0286 \%$ & $0,0559 \%$ & 75 & $2,1908 \%$ & $2,6180 \%$ \\
\hline 25 & $0,0331 \%$ & $0,0634 \%$ & 76 & $2,3469 \%$ & $2,7981 \%$ \\
\hline 26 & $0,0381 \%$ & $0,0715 \%$ & 77 & $2,5165 \%$ & $2,9945 \%$ \\
\hline 27 & $0,0436 \%$ & $0,0803 \%$ & 78 & $2,7015 \%$ & $3,2096 \%$ \\
\hline 28 & $0,0497 \%$ & $0,0898 \%$ & 79 & $2,9040 \%$ & $3,4461 \%$ \\
\hline 29 & $0,0563 \%$ & $0,1001 \%$ & 80 & $3,1263 \%$ & $3,7072 \%$ \\
\hline 30 & $0,0636 \%$ & $0,1111 \%$ & 81 & $3,3715 \%$ & $3,9970 \%$ \\
\hline 31 & $0,0715 \%$ & $0,1229 \%$ & 82 & $3,6432 \%$ & $4,3206 \%$ \\
\hline 32 & $0,0801 \%$ & $0,1356 \%$ & 83 & $3,9458 \%$ & $4,6839 \%$ \\
\hline 33 & $0,0894 \%$ & $0,1491 \%$ & 84 & $4,2848 \%$ & $5,0950 \%$ \\
\hline 34 & $0,0995 \%$ & $0,1635 \%$ & 85 & $4,6671 \%$ & $5,5637 \%$ \\
\hline 35 & $0,1104 \%$ & $0,1789 \%$ & 86 & $5,1014 \%$ & $6,1031 \%$ \\
\hline 36 & $0,1222 \%$ & $0,1953 \%$ & 87 & $5,5989 \%$ & $6,7305 \%$ \\
\hline 37 & $0,1348 \%$ & $0,2126 \%$ & 88 & $6,1745 \%$ & $7,4694 \%$ \\
\hline 38 & $0,1484 \%$ & $0,2311 \%$ & 89 & $6,8478 \%$ & $8,3523 \%$ \\
\hline 39 & $0,1629 \%$ & $0,2507 \%$ & 90 & $7,6460 \%$ & $9,4259 \%$ \\
\hline 40 & $0,1785 \%$ & $0,2714 \%$ & 91 & $8,6074 \%$ & $10,7596 \%$ \\
\hline 41 & $0,1952 \%$ & $0,2933 \%$ & 92 & $9,7873 \%$ & $12,4609 \%$ \\
\hline 42 & $0,2130 \%$ & $0,3165 \%$ & 93 & $11,2698 \%$ & $14,7065 \%$ \\
\hline 43 & $0,2320 \%$ & $0,3410 \%$ & 94 & $13,1883 \%$ & $17,8075 \%$ \\
\hline 44 & $0,2522 \%$ & $0,3669 \%$ & 95 & $15,7681 \%$ & $22,3682 \%$ \\
\hline 45 & $0,2738 \%$ & $0,3942 \%$ & 96 & $19,4225 \%$ & $29,7376 \%$ \\
\hline 46 & $0,2968 \%$ & $0,4231 \%$ & 97 & $24,9991 \%$ & $43,6671 \%$ \\
\hline 47 & $0,3212 \%$ & $0,4535 \%$ & 98 & $34,5563 \%$ & $79,9511 \%$ \\
\hline 48 & $0,3471 \%$ & $0,4856 \%$ & 99 & $54,7229 \%$ & $100,0000 \%$ \\
\hline 49 & $0,3747 \%$ & $0,5195 \%$ & 100 & $100,0000 \%$ & $100,0000 \%$ \\
\hline 50 & $0,4040 \%$ & $0,5551 \%$ & & & \\
\hline
\end{tabular}

EMPIRIA. Revista de Metodología de Ciencias Sociales. N. ${ }^{\circ}$ 13, enero-junio, 2007, pp. 143-161. ISSN: 1139-5737 
Se observa un porcentaje para las edades 0 que no parece muy razonable. De todas formas se mantiene porque el efecto de la dependencia a edades tempranas es tan poco significativo en el conjunto que no desvirtúa los resultados globales. ¡Así son los ajustes!

Las tasas de dependencia del cuadro anterior se mantienen constantes a lo largo del tiempo como hipótesis. Se sabe que algunas causas de dependencia reducen su efecto con el tiempo (p.e. la práctica generalizada de amniocentesis reduce el riesgo de síndrome de Down) pero otras aumentan (p.e. accidentes de ciclomotor entre los jóvenes). Es difícil establecer un criterio para la evolución de esta tasas ya que se carece de información histórica que nos proporcione una evolución.

\section{EVOLUCION DE LA POBLACIÓN DEPENDIENTE}

Se parte del cálculo de la tabla del año 2002, para el que se aplica la función estimada anteriormente sobre lo datos de población que se tomaron como datos de partida (año 2002), tanto para varones como para mujeres.

$$
\mathrm{D}(\mathrm{e}, \mathrm{s}, 2002)=\% \mathrm{D}_{\mathrm{es}} \times \mathrm{L}(\mathrm{e}, 2002, \mathrm{~s})
$$

Siendo:

$\mathrm{D}(\mathrm{e}, \mathrm{s}, 2002)$ : número de personas dependientes de edad e y sexo $\mathrm{s}$ en el año 2002

El resto de los años se estiman mediante la siguiente formulación:

\section{Edad cero}

Se toma como valor:

$$
\mathrm{D}(0, \mathrm{~m}, \mathrm{~s})=\% \mathrm{D}_{0 \mathrm{~s}} \times \mathrm{L}(0, \mathrm{~m}, \mathrm{~s})
$$

\section{$100>$ Edad $>0$}

$$
\begin{aligned}
& \mathrm{D}(\mathrm{e}, \mathrm{m}, \mathrm{s})=\mathrm{D}(\mathrm{e}-1, \mathrm{~m}-1, \mathrm{~s}) ? \mathrm{p}_{\mathrm{e}-1}+\mathrm{d}_{\mathrm{es}} \times \mathrm{La}(\mathrm{e}, \mathrm{m}, \mathrm{s}) \\
& \mathrm{La}(\mathrm{e}, \mathrm{m}, \mathrm{s})=\mathrm{L}(\mathrm{e}, \mathrm{m}, \mathrm{s})-\mathrm{D}(\mathrm{e}-1, \mathrm{~m}-1, \mathrm{~s}) \times \mathrm{p}_{\mathrm{e}-1}
\end{aligned}
$$

Donde:

$$
\mathrm{d}_{\mathrm{es}} \text { : Tasa de dependencia para la edad e y sexo } \mathrm{s}
$$

\section{Edad $>99$}

$$
\mathrm{D}(100, \mathrm{a}, \mathrm{s})=\mathrm{L}(100, \mathrm{a}, \mathrm{s})
$$




\section{Pirámides de edad}

Al igual que para la población total, se construyen unas pirámides de población dependiente por edades que, para ser comparables, utilizan la misma escala en el eje X y se refieren a los mismo años 2002 y 2025.
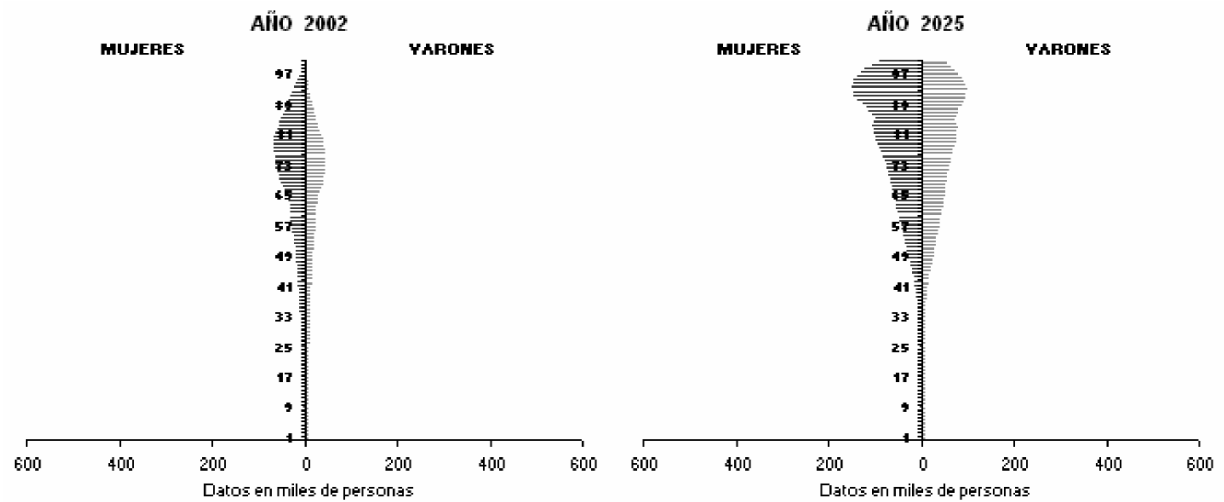

La evolución de la forma de estos gráficos da una información parcial que se completa en parte comparando la población dependiente con el total de población. Como ejemplo para estos dos mismos años:
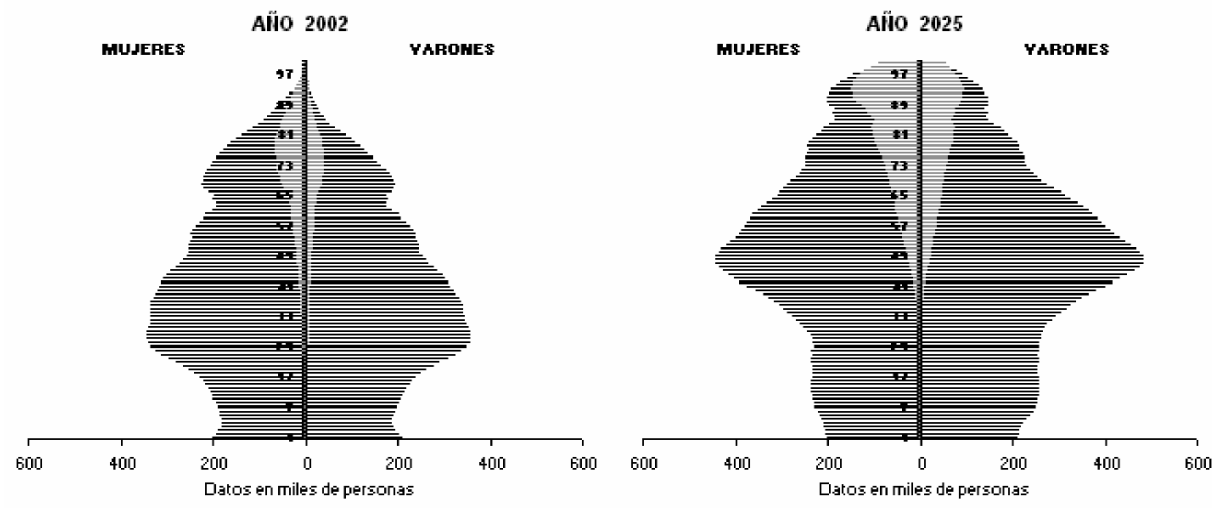

Estas pirámides se pueden expresar en forma numérica con los siguientes cuadros que agrupan resultados por tramos de edades. 
POBLACION NO DEPENDIENTE

\begin{tabular}{lrrrr}
\hline & \multicolumn{2}{c}{ AÑO 2002 } & \multicolumn{2}{c}{ AÑO 2025 } \\
\hline \multicolumn{1}{c}{ Grupo Edad } & Varones & Mujeres & Varones & Mujeres \\
\hline De 6 a 16 años & 2.303 .028 & 2.203 .136 & 2.742 .970 & 2.498 .662 \\
De 17 a 24 años & 2.357 .210 & 2.266 .588 & 2.025 .123 & 1.839 .855 \\
De 25 a 34 años & 3.422 .252 & 3.310 .557 & 2.757 .665 & 2.535 .832 \\
De 35 a 44 años & 3.060 .646 & 3.038 .804 & 3.821 .538 & 3.527 .924 \\
De 45 a 54 años & 2.363 .181 & 2.367 .301 & 4.380 .042 & 3.939 .998 \\
De 55 a 64 años & 1.763 .571 & 1.814 .859 & 3.257 .677 & 3.014 .997 \\
De 65 a 69 años & 779.220 & 846.156 & 1.135 .678 & 1.110 .557 \\
De 70 a 74 años & 624.469 & 719.667 & 886.089 & 884.478 \\
De 75 a 79 años & 404.971 & 516.694 & 719.327 & 734.920 \\
De 80 a 84 años & 191.724 & 290.067 & 497.353 & 522.310 \\
De 85 y más años & 88.974 & 162.559 & 585.063 & 616.724 \\
\hline TOTALES & 17.359 .245 & 17.536 .390 & 22.808 .526 & 21.226 .257 \\
\hline
\end{tabular}

DEPENDIENTES

\begin{tabular}{lrrrr}
\hline & \multicolumn{2}{c}{ AÑ 2002 } & \multicolumn{2}{c}{ AÑ 2025 } \\
\hline \multicolumn{1}{c}{ Grupo Edad } & \multicolumn{1}{c}{ Varones } & Mujeres & Varones & Mujeres \\
\hline De 6 a 16 años & 49.483 & 25.594 & 55.617 & 28.630 \\
De 17 a 24 años & 52.683 & 30.654 & 39.268 & 22.621 \\
De 25 a 34 años & 88.771 & 66.513 & 52.408 & 41.162 \\
De 35 a 44 años & 115.695 & 118.547 & 111.634 & 119.744 \\
De 45 a 54 años & 159.605 & 193.742 & 261.543 & 302.124 \\
De 55 a 64 años & 231.724 & 305.646 & 413.420 & 499.684 \\
De 65 a 69 años & 176.240 & 246.951 & 254.329 & 322.213 \\
De 70 a 74 años & 203.085 & 302.728 & 289.577 & 373.033 \\
De 75 a 79 años & 193.771 & 320.887 & 347.429 & 459.342 \\
De 80 a 84 años & 138.458 & 275.383 & 365.469 & 501.189 \\
De 85 y más años & 130.375 & 338.623 & 1.368 .818 & 2.178 .962 \\
\hline TOTALES & 1.539 .890 & 2.225 .267 & 3.559 .513 & 4.848 .704 \\
\hline
\end{tabular}

Si comparamos para cada tramo de edad, sexo y año el número de dependientes con la población no dependiente para ese mismo tramo de edad y sexo, obtenemos los siguientes ratios: 
PORCENTAJE DE DEPENDIENTES SOBRE NO DEPENDIENTES

\begin{tabular}{lrrrr}
\hline & \multicolumn{2}{c}{ AÑ 2002 } & \multicolumn{2}{c}{ AÑ 2025 } \\
\hline \multicolumn{1}{c}{ Grupo Edad } & Varones & Mujeres & \multicolumn{1}{c}{ Varones } & Mujeres \\
\hline De 6 a 16 años & $2,15 \%$ & $1,16 \%$ & $2,03 \%$ & $1,15 \%$ \\
De 17 a 24 años & $2,23 \%$ & $1,35 \%$ & $1,94 \%$ & $1,23 \%$ \\
De 25 a 34 años & $2,59 \%$ & $2,01 \%$ & $1,90 \%$ & $1,62 \%$ \\
De 35 a 44 años & $3,78 \%$ & $3,90 \%$ & $2,92 \%$ & $3,39 \%$ \\
De 45 a 54 años & $6,75 \%$ & $8,18 \%$ & $5,97 \%$ & $7,67 \%$ \\
De 55 a 64 años & $13,14 \%$ & $16,84 \%$ & $12,69 \%$ & $16,57 \%$ \\
De 65 a 69 años & $22,62 \%$ & $29,18 \%$ & $22,39 \%$ & $29,01 \%$ \\
De 70 a 74 años & $32,52 \%$ & $42,07 \%$ & $32,68 \%$ & $42,18 \%$ \\
De 75 a 79 años & $47,85 \%$ & $62,10 \%$ & $48,30 \%$ & $62,50 \%$ \\
De 80 a 84 años & $72,22 \%$ & $94,94 \%$ & $73,48 \%$ & $95,96 \%$ \\
De 85 y más años & $146,53 \%$ & $208,31 \%$ & $233,96 \%$ & $353,31 \%$ \\
\hline TOTALES & $8,87 \%$ & $12,69 \%$ & $15,61 \%$ & $22,84 \%$ \\
\hline
\end{tabular}

Se puede observar un fenómeno aparentemente contradictorio, ya que hasta los 69 años el porcentaje de personas dependientes sobre el total es inferior en los cálculos para el año 2025 respecto a datos del año 2002, sin embargo para edades mayores es muy superior. De hecho, el ratio global (todas las edades) para el año 2025 casi duplica el del año 2002.

Este hecho se puede explicar por efecto de la selección en la inmigración. Los inmigrantes en el momento de venir a España tienen sensiblemente menos porcentaje de personas con algún grado de dependencia que el observado para la población ya establecida. 


\title{
RESUMEN
}

La pretensión inicial es mostrar un método para estimar la evolución de una situación que tiene una relación directa importante con la edad. Nadie duda de la importancia de la medicina preventiva como complemento a la medicina curativa. Hoy en día las empresas no se venden por su valor contable o patrimonial sino por un precio que viene determinado básicamente por una estimación de su capacidad para generar beneficios futuros. Quizás la sociología se podría enriquecer con la utilización de métodos de previsión.

\section{PALABRAS CLAVE}

Dependencia, proyecciones de población, métodos de previsión.

\begin{abstract}
The initial pretension is to show a method for estimating the evolution of a situation that has had an important relation with age. Nobody doubts of the importance of preventive medicine as a complement to healing medicine. Nowadays the companies are not sold because of their wealth or countable value, but because of a price basically determined by an estimation of their capacity to generate future benefits. Maybe, Sociology could be enriched by using preventive foresight methods
\end{abstract}

\section{KEY WORDS}

Dependency, population projections, foresight methods. 